\title{
STRUCTURE SPACES AND DECOMPOSITION IN JB*-TRIPLES
}

\author{
L. J. BUNCE, C. H. CHU* and B. ZALAR
}

\section{Introduction}

Complex Banach spaces for which the group of biholomorphic automorphisms of the open unit ball acts transitively, alias $\mathrm{JB}^{*}$-triples, possess a ternary algebraic structure uniquely determined by the holomorphic properties of the open unit ball [21]. A large and important class of these spaces is comprised of the $\mathrm{JC}^{*}$-triples of [17] (known also as $J^{*}$-algebras) which are up to isometry the norm closed subspaces of $B(H, K)$, where $H$ and $K$ are complex Hilbert spaces, that are closed under the ternary product

$$
\{x y z\}=\frac{1}{2}\left(x y^{*} z+z y^{*} x\right) .
$$

Hence, $C^{*}$-algebras are $\mathrm{JC}^{*}$-triples. On the other hand, the range of contractive projection on a $C^{*}$-algebra is a $\mathrm{JC}^{*}$-triple $[13,22,27]$ but not necessarily a $C^{*}$-algebra. An "exceptional" class of JB*-triples involves certain subspaces of three by three matrices with complex Cayley numbers entries.

A detailed survey of $\mathrm{JB}^{*}$-triples recording recent developments including applications to quantum mechanics, complex holomorphy and operator algebras is to be found in [26].

Representation theory in terms of appropriate "irreducible" factors is a basic concept in algebra. In $\mathrm{JB}^{*}$-triples, for any integer $n \geq 1$, there are an infinite number of (appropriately "irreducible") Cartan factors at rank $n$. An additional complexity is the existence of six distinct generic types of Cartan factors.

The purpose of this paper is to investigate Cartan representation theory of $\mathrm{JB}^{*}$-triples. To this end we study the structure space of primitive $M$-ideals in some detail and we devise and apply techniques for decomposing $\mathrm{JB}^{*}$-triples into others with a simpler Cartan representation structure.

Received February 26, 1997; in revised form June 2, 1997. 


\section{Notation and preliminaries}

A JB*-triple is a complex Banach space $A$ with a ternary product $A^{3} \rightarrow A$ given by $(a, b, c) \mapsto\{a b c\}$ which, where $D(a, b)$ denotes the multiplication operator $x \mapsto\{a b x\}$, satisfies

(i) $\{a b c\}$ is symmetric and complex linear in $a, c$ and conjugate linear in $b$,

(ii) $[D(a, b), D(c, d)]=D(\{a b c\}, d)-D(c,\{d a b\})$,

(iii) $D(a, a)$ is hermitian with positive spectrum,

(iv) $\|\{a a a\}\|=\|a\|^{3}$.

The conjugate linear operator $x \mapsto\{a x b\}$ is denoted by $Q_{a, b}$. We write $Q_{a}=Q_{a, a}$. The elements $a, b$ are said to be orthogonal if $D(a, b)=0$ (equivalently $D(b, a)=0)$ )

A subspace $I$ of $A$ is said to be an ideal of $A$ if $\{A I A\}+\{A A I\} \subset I$ and to be an inner ideal of $A$ if $\{I A I\} \subset I$. If $I$ is a norm closed subspace, it is an ideal of $A$ if $\{A I I\} \subset I[6]$. The annihilator, $I^{\perp}=\{x:\{x I A\}=0\}$ of an ideal of $A$ is also a norm closed ideal. By [2], the norm closed ideals of $A$ are precisely the $M$-ideals.

A $\mathrm{JBW}^{*}$-triple is a $\mathrm{JB}^{*}$-triple with a (unique) predual $[2,18]$. Frequent and tacit use shall be made of the facts $[9,2]$ that the second dual $A^{* *}$ of a $\mathrm{JB}^{*}$ triple $A$ is a $\mathrm{JBW}^{*}$-triple containing $A$ as a $\mathrm{JB}^{*}$-subtriple and that the triple product is separately weak* continuous in each variable in a $\mathrm{JBW}^{*}$-triple.

Associated with a tripotent $e$ (i.e. $e=\{e e e\}$ ) in $A$ are the Peirce projections

$$
P_{2}(e)=Q_{e}^{2}, \quad P_{1}(e)=2\left(D(e, e)-Q_{e}^{2}\right), \quad P_{0}(e)=I-2 D(e, e)+Q_{e}^{2}
$$

which are mutually orthogonal with sum $I$ and ranges

$$
P_{j}(e)(A)=A_{j}(e)=\left\{x:\{e e x\}=\frac{j}{2} x\right\}
$$

giving $A=A_{2}(e) \oplus A_{1}(e) \oplus A_{0}(e)$.

$\mathrm{JB}^{*}$-algebras and their hermitian parts, $J B$-algebras, appear naturally as, for a tripotent $e$, the Peirce space $A_{2}(e)$ is a $\mathrm{JB}^{*}$-algebra with the identity $e$, product $x \circ y=\{x e y\}$ and involution $x \mapsto\{e x e\}$. If $A$ is a $\mathrm{JBW}^{*}$-triple, then $A_{2}(e)$ is a $\mathrm{JBW}^{*}$-algebra. We refer to $[15,29]$ for the theory of $\mathrm{JB}$-algebras and $\mathrm{JB}^{*}$-algebras.

The tripotent $e$ of $A$ is said to be complete if $A_{0}(e)=0$ and to be minimal if $e \neq 0$ and $A_{2}(e)=\mathrm{C} e$. For $\rho \in \partial_{e}\left(A_{1}^{*}\right)$ (extreme points of the dual ball) there is a unique minimal tripotent $e$ of $A^{* *}$ for which $\rho(e)=1$, called the support $s(\rho)$ of $\rho$. The map $\rho \mapsto s(\rho)$ is a bijection from $\partial_{e}\left(A_{1}^{*}\right)$ onto the set of minimal tripotents of a $\mathrm{JBW}^{*}$-triple $A^{* *}[12]$.

A linear bijection between $\mathrm{JB}^{*}$-triples is an isometry if and only if it is a triple homomorphism (i.e. preserves the triple product). The $\mathrm{JBW}^{*}$-triples 
containing a minimal tripotent but without proper weak* closed ideals are called Cartan factors $[8,19]$ which, up to isometry, are as follows. For arbitrary Hilbert spaces and conjugation $j: H \rightarrow H$, the $\mathrm{JB}^{*}$-triples $B(H, K)$, $\left\{x \in B(H) ; x=-j x^{*} j\right\}$ and $\left\{x \in B(H) ; x=j x^{*} j\right\}$ characterize three families of Cartan factors. A fourth is given by the complex spin factors. The remaining two exceptional Cartan factors are the $1 \times 2$ matrices over the complex octonions $\mathrm{Q}$ and the self-adjoint $3 \times 3$ matrices over $\mathrm{Q}$.

A Cartan factor $M$ is said to have infinite rank if it contains an infinite orthogonal family of tripotents. Otherwise, each maximal orthogonal family of minimal tripotents has the same finite cardinality, the rank of $M$. Apart from infinite dimensional spin factors and $B\left(H, \mathrm{C}^{n}\right)$, where $n<\infty$ and $H$ is infinite dimensional, all other finite rank Cartan factors have finite dimension.

For unmentioned and further details of $\mathrm{JB}^{*}$-triples we refer to [26, 29].

\section{Functional calculus and ideals}

In this section we show that a $\mathrm{JB}^{*}$-triple is inundated with inner ideals that are naturally $\mathrm{JB}^{*}$-algebras and we describe certain other properties of inner ideals needed later. We begin with a description of triple functional calculus.

Given an element $x$ of a $\mathrm{JB}^{*}$-triple $A$, we shall use $A_{x}$ to denote the $\mathrm{JB}^{*}$ subtriple generated by $x$. If $A$ is a $C^{*}$-algebra and $x \geq 0$, then $A_{x}$ equals the $C^{*}$-algebra generated by $x[17$, Lemma 5.7.].

On the other hand it follows from [21] that for an arbitrary JB*-triple and $x \in A$ there exists a surjective linear isometry (hence a triple isomorphism) $\varphi: A_{x} \rightarrow C$ onto a commutative $C^{*}$-algebra generated by $\varphi(x) \geq 0$. Let $\tilde{\varphi}: A_{x}^{* *} \rightarrow C^{* *}$ be the bitransposed extension of $\varphi$. In these circumstances, we shall write

$$
S(x)=\sigma(\varphi(x)) \backslash\{0\}, f_{t}(x)=\varphi^{-1} f(\varphi(x)) \text { if } f \in C_{0}(S(x)), e(x)=\tilde{\varphi}^{-1}(1),
$$

and we note that this is unambiguous. For if $\psi: A_{x} \rightarrow D$ is another surjective linear isometry onto a commutative $C^{*}$-algebra $D$ generated by $\psi(x) \geq 0$, then $\psi \varphi^{-1}: C \rightarrow D$ is a positive isometry and hence a $*$-automorphism sending $\varphi(x)$ to $\psi(x)$. So, $\left(\psi \varphi^{-1}\right)\left(f(\varphi(x))=f(\psi(x))\right.$ if $f \in C_{0}(S(x))$. Similarly, $\tilde{\psi} \tilde{\varphi}^{-1}(1)=1$. In particular,

$$
A_{x}=\left\{f_{t}(x): f \in C_{0}(S(x))\right\} .
$$

Let $A(x)$ denote the norm closure of $\{x A x\}$. Then $A(x)$ is an inner ideal of $A$, as follows from the triple identity $Q_{\{a b a\}}=Q_{a} Q_{b} Q_{a}$. With $y=\{x x x\}$ we have that $\varphi(y)=\varphi(x)^{3}$ also generates $C$ and the functional calculus gives 
$A_{x}=A_{y} \subset A(x)$. In particular, $A(x)$ is the smallest norm closed inner ideal of $A$ containing $x$ and $A(x)$ is weak* dense in $\left(A^{* *}\right)_{2}(e(x))$.

Proposition 2.1. Let $A$ be a $\mathrm{JB}^{*}$-triple and let $x \in A$. Then $A(x)$ is a $\mathrm{JB}^{*}$ subalgebra of the $\mathrm{JBW}^{*}$-algebra $\left(A^{* *}\right)_{2}(e(x))$ and contains $x$ as a positive element.

Proof. Let $\varphi: A_{x} \rightarrow C$ and its bitransition $\tilde{\varphi}: A_{x}^{* *} \rightarrow C^{* *}$ be as given above. Let $e=e(x)$ and put $y=\{x e x\}$. Then $y \in A_{x}^{* *}$ and $\tilde{\varphi}(y)=\varphi(x)^{2}$ lies in $C$ and generates it both as a $C^{*}$-algebra and as a $\mathrm{JB}^{*}$-triple. Hence, $y \in \tilde{\varphi}^{-1}(C)=A_{x}=A_{y}$. In particular, $x \in A_{y} \subset A(x)$. So, $A(y)=A(x)$.

Now let $a \in A$ and put $z=\{x a x\}$. Then

$$
\{z e z\}=Q_{x} Q_{a} Q_{x}(e)=Q_{x} Q_{a}(y) \subset A(x)
$$

and it follows that $A(x)$ is a norm closed Jordan subalgebra of $\left(A^{* *}\right)_{2}(e)$. To see that $A(x)$ is closed under involution $a \mapsto\{e a e\}$, note first that $x=\{e x e\}$ so that

$$
Q_{e}\left(Q_{y}(A)\right)=Q_{e} Q_{x} Q_{e}\left(Q_{x}(A)\right)=Q_{x}^{2}(A) \subset A(x)
$$

which gives $Q_{e}(A(x))=Q_{e}(A(y)) \subset A(x)$ and proves that $A(x)$ is a JB*-subalgebra of $\left(A^{* *}\right)_{2}(e)$. With $f(\lambda)=\lambda^{\frac{1}{2}}, \lambda \geq 0$, we have $f_{t}(x)=\left\{e f_{t}(x) e\right\}$ and $x=\left\{f_{t}(x) e f_{t}(x)\right\}$. So, $x \in A(x)_{+}$.

REMARK 2.2. (a) Let $\pi: A \rightarrow B$ be a triple homomorphism between JB*triples. Let $x \in A$ and put $y=\pi(x)$. Then it follows from the above proposition that the restriction $\pi: A(x) \rightarrow B(y)$ is a Jordan homomorphism of $\mathrm{JB}^{*}$-algebras. Further, $\pi\left(f_{t}(x)\right)=f_{t}(y)$ for all $f \in C_{0}(S(x))$.

(b) Let $A$ be a weak* dense $\mathrm{JB}^{*}$-subtriple of a $\mathrm{JBW}^{*}$-triple $M$ and let $x \in A$. Let $\pi: A^{* *} \rightarrow M$ denote the weak* continuous projection onto $M$. Put $f=\pi(e(x))$. As $\pi$ projects $\left(A^{* *}\right)_{2}(e(x))$ onto $M_{2}(f)$ and acts identically on $A(x), A(x)$ is seen to be a weak* dense $\mathrm{JB}^{*}$-subalgebra of the $\mathrm{JBW}^{*}$-algebra $M_{2}(f)$ in the obvious way.

Next we describe some relevant ideal theory of inner ideals. If $I$ is a norm closed inner ideal of a $\mathrm{JB}^{*}$-triple $A, T(I)$ shall denote the norm closed triple ideal of $A$ generated by $I$.

Lemma 2.3. Let I be a norm closed inner ideal of a $\mathrm{JB}^{*}$-triple $A$ and let $J$ be a norm closed inner ideal of I. Then $J$ is an inner ideal of $A$.

Proof. Let $x \in J$. By functional calculus, choose $y \in J$ such that $x=\{y y y\}$. Then

$$
Q_{x}(A)=Q_{y}^{2}\left(Q_{y}(A)\right) \subset Q_{y}^{2}(I) \subset J
$$


Lemma 2.4. Let A be a JB*-triple. Let I be a norm closed inner ideal of $A$ and let $J$ be a norm closed triple ideal of $A$. Then

(i) $I \cap J=\{I J I\}$,

(ii) $T(I \cap J)=T(I) \cap J$.

Proof. (i) Given $x \in I \cap J$ take $y \in i \cap J$ with $x=\{y y y\}$. Then $x \in\{I J I\}$. This gives one inclusion and the other is clear.

(ii) Suppose first that $I \cap J=0$. Given $x \in I$ and $y \in J$ we have $Q_{x} Q_{y}=0$ so that $Q_{\{y x y\}}=Q_{y} Q_{x} Q_{y}=0$ implying that $\{J I J\}=0$. By the fundamental identity

$$
\{J\{J I I\} J\} \subset\{\{J J J\} I J\}-\{J J\{J I J\}\}=0
$$

which gives $\{J J I\}=0$. In turn, we have

$$
\{\{I J A\} J J\}=\{I J\{A J J\}-\{A J\{I J J\} J\}=0 .
$$

So, $\{I J A\}=0$ giving $I \subset J^{\perp}$ and so $T(I) \subset J^{\perp}$. Hence, $T(I) \cap J=0$.

Reverting to the general case, the canonical surjection $\pi: A \rightarrow A / T(I \cap J)$ gives $\pi(T(I))=T(\pi(I))$ and $\{\pi(I) \pi(J) \pi(I)\}=\pi(\{I J I\})=0$. Therefore, by (i) together with the first part of the proof of (ii),

$$
\pi(T(I) \cap J)=T(\pi(I)) \cap \pi(J)=0
$$

Hence, $T(I) \cap J \subset T(I \cap J)$, as required.

Proposition 2.5. Let I be a norm closed inner ideal of a $\mathrm{JB}^{*}$-triple $A$ and let $J$ be a norm closed triple ideal of $I$. Then $J=T(J) \cap I$.

Proof. Let $f$ be a complete tripotent of $I^{* *}$ and, via [18, (4.2)], let $e$ be a complete tripotent of $A^{* *}$ such that $f$ is a projection of the $\mathrm{JBW}^{*}$-algebra $M=\left(A^{* *}\right)_{2}(e)$. Now, $J^{* *} \cap N$ is a weak* closed Jordan ideal of the hereditary $\mathrm{JBW}^{*}$-subalgebra $N=\{f M f\}$ of $M$. Thus, by [11, Theorem], there is a central projection $z$ of $M$ such that $J^{* *} \cap N=z \circ N$, where $\circ$ dentoes the Jordan product in $M$. In particular, $J^{* *} \cap N$ is contained in the weak* closed triple ideal of $A^{* *}, K=\left(A^{* *}\right)_{2}(z)+\left(A^{* *}\right)_{1}(z)$, and so lies in $\left(K \cap I^{* *}\right) \cap N$. By [18, (4.2)] applied to $I^{* *}$ this gives $J^{* *} \subset K \cap I^{* *}$ from which it follows that $T(J)^{* *} \subset K$. Hence,

$$
T(J)^{* *} \cap N \subset K \cap N=(z \circ M) \cap N \subset z \circ N=J^{* *} \cap N .
$$

But then $\left(T(J)^{* *} \cap I^{* *}\right) \cap N=J^{* *} \cap N$ so that, as before, [17, (4.2)] gives $T(J)^{* *} \cap I^{* *}=J^{* *}$. Intersecting both sides of which with $A$ results in $T(J) \cap I=J$. 


\section{The structure space}

The structure space of primitive $M$-ideals of a Banach space was introduced and investigated in [1]. A particularly important and very comprehensive reference on $M$-ideals is given by [16] to which we refer, together with [4], for any unmentioned details or $M$-structure in Banach spaces.

It was shown in [2] that the $M$-ideals of a $\mathrm{JB}^{*}$-triple $A$ are the norm closed ideals. By a primitive ideal of $A$ we shall mean primitive $M$-ideal. Thus $P$ is primitive ideal of $A$ if for some $\rho \in \partial_{e}\left(A_{1}^{*}\right)$ is the largest norm closed ideal of $A$ contained in $\operatorname{ker} \rho$. Let $\operatorname{Prim}(A)$ denote the set of all primitive ideals of $A$ and given $X \subset A, S \subset \operatorname{Prim}(A)$ write

$$
h(X)=\{P \in \operatorname{Prim}(A): X \subset P\}, k(S)=\cap\{P \in \operatorname{Prim}(A): P \in S\} .
$$

Primitive ideals are prime ideals (in the usual sense) and there is a unique topology on $\operatorname{Prim}(A)$, the structure topology, for which $h k(S)$ is the closure of $S$. Endowed with this structure topology, $\operatorname{Prim}(A)$ is referred to as the structure space of $A$. There is a bijective correspondance, $J \rightarrow h(J)$, between the norm closed ideals of $A$ and the closed sets of $\operatorname{Prim}(A)$ and we have the homeomorphisms

$$
h(J) \rightarrow \operatorname{Prim}(A / J)
$$

and

$$
\operatorname{Prim}(A) \backslash h(J) \rightarrow \operatorname{Prim}(J) \quad(P \mapsto P \cap J)
$$

for each norm closed ideal $J$ of $A$.

A triple homomorphism, $\pi: A \rightarrow M$, into a $\mathrm{JBW}^{*}$-triple $M$ has unique weak* continuous extension, $\tilde{\pi}: A^{* *} \rightarrow M$, with $\tilde{\pi}:\left(A^{* *}\right)=\overline{\pi(A)}$ [3], where here and later the bar refers to weak* closure. If $M$ is a Cartan factor and $\overline{\pi(A)}=M$, then $\pi$ is said to be a Cartan factor representation. The set of all Cartan factor representations of $A$ is denoted by $C(A)$.

Given $\rho \in \partial_{e}\left(A_{1}^{*}\right)$, let $A_{\rho}^{* *}$ be the weak* closed ideal of $A^{* *}$ generated by the (minimal) support tripotent $s(\rho)$ [12]. Then $A_{\rho}^{* *}$ is a complemented Cartan factor in $A^{* *}[8,18]$ and the restriction, $\pi_{\rho}: A \rightarrow A_{\rho}^{* *}$, of the natural weak* continuous projection, $P_{\rho}: A^{* *} \rightarrow A_{\rho}^{* *}$, is a Cartan factor representation of $A$.

The following is contained in detail of [2, Theorem 3.6].

Lemma 3.1. Let $A$ be a $\mathrm{JB}^{*}$-triple and let $\rho$ be an extreme point of the dual ball. Then $\operatorname{ker} \pi_{\rho}$ is the largest norm closed ideal of $A$ in $\operatorname{ker} \rho$. Hence, $\operatorname{Prim}(A)=\left\{\operatorname{ker} \pi_{\rho}: \rho \in \partial_{e}\left(A_{1}^{*}\right)\right\}$.

Lemma 3.2. Let $\pi: A \rightarrow M$ be a Cartan factor representation of a $\mathrm{JB}^{*}$-triple 
A. Then there exists $\rho \in \partial_{e}\left(A_{1}^{*}\right)$ and a surjective isometry $\varphi: A_{\rho}^{* *} \rightarrow M$ such that $\varphi \pi_{\rho}=\pi$. Hence, $\operatorname{Prim}(A)=\{\operatorname{ker} \pi: \pi \in C(A)\}$.

Proof. Let $J=\operatorname{ker} \tilde{\pi}$ where $\tilde{\pi}: A^{* *} \rightarrow M$ is a weak* continuous extension of $\pi$ onto $M$. Then the complement of $J$ in $A^{* *}, J^{\perp} \approx A^{* *} / J \approx M$. Choose a minimal tripotent $e$ of $A^{* *}$ contained in $J^{\perp}$ and let $\rho \in \partial_{e}\left(A_{1}^{*}\right)$ with $s(\rho)=e$, using [12, Proposition 4]. It follows that $A_{\rho}^{* *}=J^{\perp}$ and that $\varphi \pi_{\rho}=\pi$.

Proposition 3.3. Let I be a norm closed inner ideal of a $\mathrm{JB}^{*}$-triple A. Then

$$
\beta: \operatorname{Prim}(A) \backslash h(I) \rightarrow \operatorname{Prim}(I) \quad(P \mapsto P \cap I)
$$

is a homeomorphism.

Proof. As a weak* closed inner ideal of a Cartan factor is a Cartan factor, it follows that a Cartan factor representation of $A$ which fails to kill $I$ restricts to a Cartan factor representation of $I$. It follows from Lemma 3.2 that $\beta$ is well-defined.

On the other hand, given $\rho \in \partial_{e}\left(I_{1}^{*}\right)$ let $\bar{\rho} \in \partial_{e}\left(A_{1}^{*}\right)$ extend $\rho$. As $s(\rho)$ is minimal in the weak* closed inner ideal $I^{* *}$ it is also minimal in $A^{* *}$. So $s(\rho)=s(\bar{\rho})$ and hence, $I_{\rho}^{* *} \subset A_{\bar{\rho}}^{* *}$. Let $G$ be the complementary ideal of $I_{\rho}^{* *}$ in $I^{* *}$. Let $J$ be the norm closed ideal generated by in $I_{\rho}^{* *}$ in $A_{\bar{\rho}}^{* *}$. Then $G \subset J^{\perp}$ by Lemma 2.4. Hence, $G \subset A_{\bar{\rho}}^{* *}$ because $J^{\perp}=(\bar{J})^{\perp}=A_{\bar{\rho}}^{* *}$. It follows that $P_{\bar{\rho}}: A^{* *} \rightarrow A_{\bar{\rho}}^{* *}$ restricts to $P_{\rho}: I^{* *} \rightarrow I_{\rho}^{* *}$ so that $\pi_{\bar{\rho}}$ restricts to $\pi_{\rho}$ and hence $\operatorname{ker} \pi_{\rho}=I \cap \operatorname{ker} \pi_{\bar{\rho}}$. Therefore, $\beta$ is surjective by Lemma 3.1.

By Lemma 2.4 together with primeness of primitive ideals, $\beta$ is injective and for each norm closed ideal $J$ of $A, \beta(h(J) \backslash h(I))=h(I \cap J)$ (taken in $I$ ) so that $\beta$ is a closed map. By Proposition 2.5, the right hand side of the equation runs through all closed sets of $\operatorname{Prim}(I)$ and so $\beta$ is continuous.

Remark 3.4. Let $A$ be a JB*-algebra, $\pi: A \rightarrow M$ a Cartan factor representation and $\tilde{\pi}: A^{* *} \rightarrow M$ its weak* continuous extension. Then with $e=\tilde{\pi}(1), \pi$ is reconstituted as a * Jordan Cartan factor representation, $\pi: A \rightarrow M_{2}(e)(=M)$ and induces by restriction (in the sense of $15, \mathrm{p} .133$ ) a Jordan type I factor representation of the JB-algebra $A_{\mathrm{sa}}$. Thus, by restriction and by complexification in the opposite direction (cf. [29]) the structure space of $A$ is naturally identified with the usual structure space [6] of the JCalgebra $A_{\mathrm{sa}}$. We shall make frequent and often tacit use of this fact.

Lemma 3.5. Let $A$ be a $\mathrm{JB}^{*}$-triple.

(i) $\hat{x}: \operatorname{Prim}(A) \mapsto[0, \infty)(P \mapsto\|x+P\|)$ is lower semicontinuous for all $x$.

(ii) The sets $\{P \in \operatorname{Prim}(A):\|x+P\| \geq \alpha\}$, where $\alpha>.0$ and $x \in A$, form a basis of quasi-compact sets for $\operatorname{Prim}(A)$.

(iii) $\operatorname{Prim}(A)$ is Hausdorff if $\hat{x}$ and only if is continuous for all $x \in A$. 
Proof. When $A$ is a $\mathrm{JB}^{*}$-algebra and " $x \in A$ " is replaced by " $x \in A_{+}$", (i), (ii) and (iii) follow as for $C^{*}$-algebras (cf. [10, (3.3)], [25, (4.4)].

(i): Let $x \in A$. Via the triple and hence isometric embedding $A(x) / P \cap A(x) \rightarrow A / P$ we have $\|x+P\|=\|x+P \cap A(x)\|$ for each $P \in \operatorname{Prim}(A)$. Consider the open set $U=\operatorname{Prim}(A) \backslash h(A(x)) \approx \operatorname{Prim}(A(x))$. By Proposition 2.1., $A(x)$ can be realised as a $\mathrm{JB}^{*}$-algebra such that $x$ is positive there. Therefore, the opening remark together with Proposition 3.3 imply that $\hat{x}: U \rightarrow[0, \infty)$ is lower semicontinuous. But for $\alpha \geq 0, \hat{x}^{-1}((\alpha, \infty))$ is contained in $U$ by Proposition 3.3. Hence, $\hat{x}$ is lower semicontinuous on $\operatorname{Prim}(A)$.

(ii), (iii): Via the opening remark these follow by similar use of Proposition 2.1 and Proposition 3.3.

\section{Rank and collinear systems}

Let $A$ be a JB*-triple. The $\operatorname{rank}, \operatorname{rank}(\pi)$, of a Cartan factor representation, $\pi: A \rightarrow M$, is the rank of $M$. If for fixed $n$, where $1 \leq n<\infty, \operatorname{rank}(\pi)=n$ for all Cartan representations, then $A$ is said to be of constant rank $n$. The $\mathrm{JB}^{*}$-triple is said to be of bounded $\operatorname{rank}$ if $\{\operatorname{rank}(\pi): \pi \in C(A)\}$ is bounded.

In the Cartan factor $M$, the $\mathrm{JB}^{*}$-subtriple generated by all minimal tripotents is a simple norm closed ideal, $K(M)$, of $M$ such that its second dual is isometric to $M$ [7]. We have,

$M$ has finite rank if and only if $M$ is reflexive if and only if $K(M)=M$.

As seen in the proof of Proposition 3.3., given $\pi \in C(A)$ and $x \in A$ with $\pi(x) \neq 0, \pi$ restricts to a Cartan factor representation of $A(x)$. In the following this induced representation is denoted by $\pi_{x}$.

Lemma 4.1. Let $A$ be a $\mathrm{JB}^{*}$-triple and $\pi: A \rightarrow M$ a Cartan representation of $A$.

(i) If $\operatorname{rank}(\pi)<\infty$, then there exists $x \in A$ with $\pi(x) \neq 0$ such that $\operatorname{rank}(\pi)=\operatorname{rank}\left(\pi_{x}\right)$.

(ii) If for all $x \in A$ with $\pi(x) \neq 0$ we have $\operatorname{rank}\left(\pi_{x}\right)<\infty$, then $\operatorname{rank}(\pi)<\infty$.

Proof. (i): Suppose that the Cartan factor has a finite rank. Then $M$ is reflexive so that $\pi(A)=M$. Choose $x \in A$ such that $\pi(x)=e$ is a complete tripotent of $M$. Then $\pi_{x}(A(x))=M_{2}(e)$. Hence, $\operatorname{rank}\left(\pi_{x}\right)=\operatorname{rank}(\pi)$.

(ii): Let $x \in A$ be such that $\pi(x) \neq 0$ and $\operatorname{rank}\left(\pi_{x}\right)<\infty$. Then $\pi(A(x))=\pi_{x}(A(x))$ is a reflexive, so weak* closed, inner ideal of $M$ which implies that $\pi(A(x)) \subset K(M)$. Hence, given that the stated condition is satisfied, $\pi(A) \subset K(M)$. Now, the natural projection $Q: K(M)^{* *} \rightarrow M$ is an 
isometry onto $M$ and $Q$ maps $\pi(A)^{* *}$ onto $\overline{\pi(A)}=M$. Therefore, $\pi(A)^{* *}=K(M)^{* *}$ and so $\pi(A)=K(M)$. It follows that $\pi$ has a finite rank. Otherwise, there is an infinite sequence $\left(e_{n}\right)$ of orthogonal minimal tripotents in $M$. In this case $y=\sum \frac{e_{n}}{2^{n}} \in K(M)$ and, choosing $x \in A$ with $\pi(x)=y$ and putting $e=\sum e_{n}$, we obtain that $\pi_{x}: A_{x} \rightarrow M_{2}(e)$ is a Cartan factor representation of infinite rank. This contradiction concludes the proof.

For a $\mathrm{JB}^{*}$-triple $A$ and natural number $n$ we denote by $\operatorname{Prim}_{n}(A)$ the set of those primitive ideals ker $\pi$ for which $\operatorname{rank}(\pi) \leq n$.

Proposition 4.2. Let $A$ be a $\mathrm{JB}^{*}$-triple and $n$ a natural number. Then $\operatorname{Prim}_{n}(A)$ is closed in $\operatorname{Prim}(A)$.

Proof. Take $\pi \in C(A)$ such that $\operatorname{ker} \pi \notin \operatorname{Prim}_{n}(A)$. By Lemma 4.1 there exists $x \in A$ such that $\pi(x) \neq 0$ and ker $\pi \cap A(x)=\operatorname{ker} \pi_{x} \notin \operatorname{Prim}_{n}(A(x))=$ $F$, which is closed in $\operatorname{Prim}(A(x))$ as follows from Proposition 2.1 together with [5, Lemma 6]. Now $U=\beta^{-1}(\operatorname{Prim}(A(x)) \backslash F)$, where $\beta$ is the homeomorphism of Proposition 3.3, satisfies $U \cap \operatorname{Prim}_{n}(A)=\emptyset$, and $U$ is an open neighbourhood of ker $\pi$. This proves that $\operatorname{Prim}_{n}(A)$ is closed.

Remark 4.3. Given a $\mathrm{JB}^{*}$-algebra $A$ consider the functions $T_{x}: \operatorname{Prim}(A) \rightarrow[0, \infty], \quad x \in A_{+}^{* *}, \quad$ given $\quad$ by $\quad T_{x}(\operatorname{ker} \pi)=\operatorname{Tr}(\tilde{\pi}(x))$ where $\pi: A \rightarrow M$ and $\tilde{\pi}: A^{* *} \rightarrow M$ is its weak* continuous extension and $\operatorname{Tr}$ is the Jordan trace on $M$. The functions $T_{x}$ are lower semicontinuous for all $x \in A_{+}$(cf. [5, Lemma 6]. Hence, $T_{x}$ is lower semicontinuous whenever $x \in A^{* *}$ is the strong limit of an increasing net in $A_{+}$. If $A$ has constant rank $n$, then it follows as for $C^{*}$-algebras (cf. $[25,4.4 .10]$ that $T_{x}$ is continuous for all $x \in A_{+}$and that $\operatorname{Prim}(A)$ is Hausdorff.

Lemma 4.4. Let $A$ be a $\mathrm{JB}^{*}$-triple of constant finite rank $n$. Then $\operatorname{Prim}(A)$ is Hausdorff.

Proof. Let $P_{1}, P_{2} \in \operatorname{Prim}(A)$ with $P_{1} \neq P_{2}$. By assumption, the canonical maps $\pi_{i}: A \rightarrow A$ ? $P_{i}=M_{i}$ belong to $C(A) i=1,2$. For $i=1,2$, choose $x_{i} \in A$ such that $\pi_{i}\left(x_{i}\right)=e_{i}$ is a complete tripotent of $M_{i}$ and let $a_{i} \in P_{i}$ such that $x_{i}-x_{2}=a_{1}+a_{2}$, which is possible because $P_{1}+P_{2}=A$. For $x=x_{1}-a_{1}=$ $x_{2}+a_{2}$ we have $\pi_{1}(x)=e_{1}, \quad \pi_{2}(x)=e_{2}$. Hence, for $i=1,2$, the $Q_{i}=P_{i} \cap A(x)$ are, by Proposition 3.3, distinct elements of

$$
\operatorname{Prim}_{n}(A(x)) \backslash \operatorname{Prim}_{n-1}(A(x))=\operatorname{Prim}(A(x)) \backslash h(J) \approx \operatorname{Prim}(J),
$$

where $J$ is the closed ideal of $A(x)$ with hull equal to $\operatorname{Prim}_{n-1}(A(x))$ (where we let $J=0$ if $n=1$ ). But then $J$ is a $\mathrm{JB}^{*}$-algebra of constant rank (using Proposition 2.1) so that $\operatorname{Prim}(J)$ is Hausdorff by Remark 4.3. Now Proposi- 
tion 3.3 implies that $P_{1}, P_{2}$ are separated by open sets in $\operatorname{Prim}(A) \backslash h(A(x))$. Hence $\operatorname{Prim}(A)$ is Hausdorff.

In the following, which is inspired by [28, pp. 506-507], we let $h$ be the continuous function on $\mathrm{R}$ satisfying

$$
h\left(\left(-\infty, \frac{1}{4}\right)\right)=\{0\}, h\left(\left[\frac{3}{4}, \infty\right)\right)=\{1\} \text { and } h \text { is linear on }\left[\frac{1}{4}, \frac{3}{4}\right] .
$$

Recall that $h_{t}(x)$ refers to the element of $A_{x}$ given by the triple functional calculus (see $\S 2$ ). We shall also use the following: given tripotents $e$ and $f$ in a $\mathrm{JBW}^{*}$-triple such that $e$ is minimal and $\|e-f\|<1$, it follows that $f$ is minimal too. For, indeed, $P_{2}(f)(e)=\alpha u$ where $u$ is a minimal tripotent and $\alpha \in \mathrm{C}\left[12\right.$, Proposition 6] so that $\|f-\alpha u\|=\left\|P_{2}(f)(f-e)\right\|<1$ which implies that $u$ is invertible in the $\mathrm{JBW}^{*}$-algebra $M_{2}(f)$. Hence, $M_{2}(f)=M_{2}(u) \simeq \mathrm{C}$.

Lemma 4.5. Let $A$ be a $\mathrm{JB}^{*}$-triple of constant finite rank $n$. Let $P_{0} \in \operatorname{Prim}(A)$ and let $x \in A$ such that $x+P_{0}$ is a nonzero tripotent.

(i) $h_{t}(x)+P_{0}=x+P_{0}$ and $h_{t}(x)+P$ is a nonzero tripotent for all $P$ in some neighbourhood $V$ of $P_{0}$.

(ii) If $x+P_{0}$ is minimal, then $h_{t}(x)+P$ is a minimal tripotent for all $P$ in some neighbourhood $W$ of $P_{0}$.

Proof. (i) Regarding $A(x)$ as a JB*-algebra and $x \in A(x)_{+}$by Proposition 2.1, we have that $x+Q_{0}$ is a non-zero projection in $A(x) / Q_{0}$ where $Q_{0}=P_{0} \cap A(x)$. As $\operatorname{Prim}(A)$ is Hausdorff by Lemma 4.4, so $\operatorname{Prim}(A(x))$ is Hausdorff by Proposition 3.3, and the argument on page 506 of [28] gives an open neighbourhood $V$ of $Q_{0}$ such that $h(x)+Q_{0}=x+Q_{0}$ and $h(x)+Q$ is a non-zero projection for all $Q \in V$. Now Proposition 3.3 together with triple functional calculus gives (i).

(ii) Let $\pi_{0}: A \rightarrow A / P_{0}=M$ be the quotient map and let $\pi_{0}(x)=e$ be a minimal tripotent of $M$. Choose a complete tripotent $u$ of $M$ such that the Type $I_{n} \mathrm{JBW}^{*}$-factor $M_{2}(u)$ contains $e$ as a minimal projection (cf [18]). Choose $y \in A$ with $\pi(y)=u$ and let $I$ be the norm closed ideal of the $\mathrm{JB}^{*}$ algebra $B=A(y)$ corresponding to $\operatorname{Prim}(B) \backslash \operatorname{Prim}_{n-1}(B)$. Then $J$ is a JB*-algebra of constant rank $n$ and $P_{0} \cap J \in \operatorname{Prim}(J)$. Choose with $z \in J$ with $z \geq 0$ and $\pi_{0}(z)=e$. Transparent modification to the argument on page 507 of [28] now gives that $h(z)+Q$ is a minimal projection in $J / Q$ for all $Q$ in a neighbourhood of $P_{0} \cap J$ in $\operatorname{Prim}(J)$. Via Proposition 3.3., this gives rise to a neighbourhood $U$ of $P_{0}$ in $\operatorname{Prim}(A)$ such that $h_{t}(x)+P$ is a minimal tripotent for all $P \in U$. We may suppose that $U \subset V$, where $V$ is given in (i). Now put $W=\left\{P \in U:\left\|h_{t}(x)-h_{t}(z)+P\right\|<1\right\}$. Then $P_{0} \in W$ and $W$ is open by 
Lemma 3.5 (iv) and Lemma 4.4. Finally, by the remark immediately preceding the statement, $h_{t}(x)+P$ is minimal for all $P \in W$.

Tripotents $e$ and $f$ in a $\mathrm{JB}^{*}$-triple $A$ are said to be collinear if $e \in A_{1}(f)$ and $f \in A_{1}(e)$. If $e_{1}, \ldots, e_{n}$ are minimal and mutually collinear in $A$, we say that they form a collinear system of length $n$.

Lemma 4.6. Let $A$ be a $\mathrm{JB}^{*}$-triple.

(i) If $e$ and $f$ are minimal tripotents in $A$ and $e \in A_{1}(f)$, then $f \in A_{1}(e)$.

(ii) If $e_{1}, \ldots, e_{n}$ is a collinear system in $A$ and

$$
T=\left(1-\sum_{i=1}^{n} P_{2}\left(e_{i}\right)\right)\left(I-P_{0}\left(e_{1}\right)\right) \ldots\left(I-P_{0}\left(e_{n}\right)\right),
$$

then $T(A) \subset \bigcap_{i=1}^{n} A_{1}\left(e_{i}\right)$.

Proof. (i) This follows from [12, Lemma 2.1].

(ii) Let $e_{1}, \ldots, e_{n}$ be mutually collinear minimal tripotents. Put $a=y-\sum_{i=1}^{n} P_{2}\left(e_{i}\right) y$ where $y=\left(i-P_{0}\left(e_{1}\right)\right) \ldots\left(I-P_{0}\left(e_{n}\right)\right)(x)$ and $x \in A$. The Peirce projections $P_{k}\left(e_{i}\right), k=0,1,2, i=1, \ldots, n$, commute by [18, (1.10)], so $P_{0}\left(e_{j}\right) y=0, j=1, \ldots, n$ and

$$
\begin{aligned}
2 D\left(e_{j}, e_{j}\right)(a) & =2 D\left(e_{j}, e_{j}\right) y-\sum_{i \neq j} P_{2}\left(e_{i}\right) y-2 P_{2}\left(e_{j}\right) y \\
& =\left(2 D\left(e_{j}, e_{j}\right)-P_{2}\left(e_{j}\right)-I\right) y+\left(y-\sum_{i=1}^{n} P_{2}\left(e_{i}\right) y\right) \\
& =-P_{0}\left(e_{j}\right)(y)+a \\
& =a .
\end{aligned}
$$

Proposition 4.7. Let $A$ be a $\mathrm{JB}^{*}$-triple of constant rank and $m$ a natural number. Then the set

$$
S=\{P \in \operatorname{Prim}(A): A / P \text { contains a collinear system of length }>m\}
$$
is open in $\operatorname{Prim}(A)$.

Proof. Let $P_{0} \in S$. By assumption, $A / P_{0}$ contains minimal and mutually collinear tripotents $e_{1}, \ldots, e_{n}$, where $n>m$. Choose $x_{1}, \ldots, x_{n} \in A$ such that $x_{i}+P_{0}=e_{i}, i=1, \ldots, n$.

We shall show, by induction, that for all $P$ in some neighbourhood of $P_{0}, A / P$ contains a collinear system of length $n$. To this end we make the following induction hypothesis. 
Let $1 \leq k<n$ and suppose that we have $y_{1}, \ldots, y_{k} \in A$ and a neighbourhood $U$ of $P_{0}$ such that $y_{i}+P_{0}=e_{i}, i=1, \ldots, k$, and $\left\{y_{1}+P, \ldots, y_{k}+P\right\}$ is a collinear system for all $P \in U$. By Lemma 4.5, we note that this hypothesis holds for $k=1$. Put

$$
y=\left(I=-\sum_{i=1}^{k} Q\left(y_{1}\right)^{2}\right)\left(2 D\left(y_{1}, y_{1}\right)-Q\left(y_{1}\right)^{2}\right) \ldots\left(2 D\left(y_{k}, y_{k}\right)-Q\left(y_{k}\right)^{2}\right)\left(x_{k+1}\right)
$$

and $y_{k+1}=h_{t}(y)$, where $h$ is the function defined prior to Lemma 4.5. Then,

$$
y+P_{0}=\left(I-\sum P_{2}\left(e_{i}\right)\right)\left(I-P_{0}\left(e_{i}\right)\right) \ldots\left(I-P_{0}\left(e_{k}\right)\right)\left(e_{k+1}\right)=e_{k+1} .
$$

Hence, by Lemma 4.5, $y_{k+1}+P_{0}=e_{k+1}$ and there is a neighbourhood $V$ of $P_{0}$ such that

$$
y_{k+1}+P \text { is a minimal tripotent for all } P \in V .
$$

Also, by Lemma 4.6(ii) we have, for all $P \in U \cap V=W$,

$$
y+P \in \bigcap_{i=1}^{k}(A / P)_{1}\left(y_{i}+P\right) \text { so that } y_{k+1}+P \in \bigcap_{i=1}^{k}(A / P)_{1}\left(y_{i}+P\right),
$$

(as latter is a $\mathrm{JB}^{*}$-subtriple of $A / P$ ) and hence $\left\{y_{1}+P, \ldots, y_{k+1}+P\right\}$ is a collinear system by $(\alpha)$ together with Lemma 4.6(i). This completes the proof.

Elements $a, b$ in a $\mathrm{JC}^{*}$-algebra are said to be J-orthogonal if $L_{a}(b)+\frac{1}{2}(a b+b a)=0$. Let $V_{\alpha}$ be the spin factor that, when realised as a $\mathrm{JC}^{*}$-algebra, contains a maximal $J$-orthogonal family of symmetries $\left\{s_{i}\right\}_{i \in I}$ with $\operatorname{card}(I)=\alpha(\operatorname{cf}$. [15, Chapter 6]).

Lemma 4.8. Let $V$ be a spin factor realised as a $\mathrm{JC}^{*}$-algebra.

(i) If $s$, $t$ are $J$-orthogonal symmetries, then $L_{s}^{2} L_{t}^{2}=L_{t}^{2} L_{s}^{2}$.

(ii) If $s_{1}, \ldots, s_{n}$ are mutually $J$-orthogonal symmetries in $V$, then $\left(I-L_{S_{1}}^{2}\right) \ldots\left(I-L_{s_{n}}^{2}\right)(V)$ is elementwise $J$-orthogonal to $s_{i}$ for all $i=1, \ldots, n$.

(iii) Let $x^{*}=x \in V$ be nonzero and J-orthogonal to a symmetry in $V$. Then each nontrivial symmetry in the JC-algebra generated by $x$ (there are two) is a scalar multiple of $x$.

Proof. (i) is routine and (ii) then follows from the rule $L_{s_{i}}^{3}=L_{s_{i}}$.

(iii) Let $t \neq \pm 1$ be a symmetry $J$-orthogonal to $x=\alpha \cdot 1+\beta s$ where $\alpha, \beta \in \mathbf{R}$ and $s$ is a nontrivial symmetry. We have $L_{t}(s) \in \mathbf{R}$ so that $\alpha=0$. The JC-algebra generated by $x$ is $\{\lambda \cdot 1+\mu s: \lambda, \mu \in \mathrm{R}\}$, the only nontrivial symmetries in which are $s$ and $-s$

For a $\mathrm{JB}^{*}$ triple $A$ and integer $n \geq 2$, let 


$$
S^{n}(A)=\left\{P \in \operatorname{Prim}(A): A / P \approx V_{m}, m>n\right\} .
$$

Lemma 4.9. Let $A$ be a $\mathrm{JB}^{*}$-algebra for which all Cartan factor representations have rank 1 or 2 and let $n$ be an integer with $n \geq 2$. Then $S^{n}(A)$ is open in $\operatorname{Prim}(A)$.

Proof. As $S^{n}(A) \subset \operatorname{Prim}(A) \backslash \operatorname{Prim}_{1}(A)$ we may suppose that $A$ has constant rank 2. Let $h$ be the real function given prior to Lemma 4.5 and let $f, g: \mathrm{R} \rightarrow \mathrm{R}$ be given by $f(\lambda)=\frac{\lambda(\lambda+1)}{2}, g(\lambda)=h(f(\lambda))-h[(1-h(f(\lambda)))$ $\cdot(f(-\lambda))]$.

Let $P_{0} \in \operatorname{Prim}(A)$. Let $x \in A_{\mathrm{sa}}$ such that $x+P_{0}=s$ is a non-trivial symmetry in $A / P_{0}$. Then with $x_{1}=f(x)$ and $x_{2}=f(-x)$ we have $x_{1}+P_{0}=e_{1}, x_{2}+P_{0}=e_{2}$ are orthogonal minimal projections in $A / P_{0}$ with sum unity. So (cf. [27, pages 506-507] or Lemma 4.5) with $y_{1}=h\left(x_{1}\right), y_{2}=h\left(\left(1-y_{1}\right) x_{2}\right)$ we have that, $y_{1}+P_{0}=e_{1}, y_{2}+P_{0}=e_{2}$ and $y_{1}+P, y_{2}+P$ are orthogonal minimal projections in $A / P$ for all $P$ in a neighbourhood $U$ of $P_{0}$. Note that $g(x)=y_{1}-y_{2}$. Hence, $g(x)+P_{0}=s$, and $g(x)+P$ is a non-trivial symmetry for all $P \in U$.

Now suppose that $P_{0} \in S^{n}(A)$. Then, for some $m>n$, there exist $x_{1}, \ldots, x_{m}$ in $A_{\mathrm{sa}}$ such that $x_{1}+P_{0}=s_{1}, \ldots, x_{m}+P_{0}=s_{m}$ are mutually $J-$ orthogonal symmetries in $A / P_{0}$. We proceed by induction to show that there exist $y_{1}, \ldots, y_{m} \in A_{\mathrm{sa}}$ such that $y_{1}+P, \ldots, y_{m}+P$ are mutually $J$-orthogonal symmetries in some neighbourhood of $P_{0}$.

Let $1 \leq k<m$. Suppose that $y_{1}, \ldots, y_{k} \in A_{\mathrm{sa}}$ have been chosen so that $y_{i}+P_{0}=s_{i}, i=1, \ldots, k$ and $y_{1}+P, \ldots, y_{K}+P$, are mutually orthogonal symmetries for all $P$ in a neighbourhood $V$ of $P_{0}$.

Put $y=\left(I-L_{y_{1}}^{2}\right) \ldots\left(I-L_{y_{k}}^{2}\right)\left(x_{k+1}\right)$ and put $y_{k+1}=g(y)$. Then, by the first part of the proof, $y_{k+1}+P_{0}=s_{k+1}$ and $y_{k+1}+P$ is a non-trivial symmetry in $A / P$ for all $P$ in a neighbourhood $W$ of $P_{0}$. It follows from Lemma 4.8 (ii, iii), that $y_{1}+P, \ldots, y_{k+1}+P$ are mutually $J$-orthogonal symmetries in $A / P$ for all $P \in V \cap W$. Hence, $y_{1}, \ldots, y_{k+1}$ satisfy the inductive hypothesis and the result follows.

\section{Decompositions of $\mathrm{JB}^{*}$-triples}

We apply the structure space techniques developed earlier to study decomposition in $\mathrm{JB}^{*}$-triples. We are mostly interested in $\mathrm{JB}^{*}$-triples of bounded rank. Some results are more general. Relevant features and notation of finite rank Cartan factors are listed below for convenience. There are six generic types (cf. [17, 24]).

(1) Rectangular: $M_{n, \alpha}=B(H, K), 1 \leq n=\operatorname{dim}(K) \leq \alpha=\operatorname{dim}(H), n<\infty$ $(n \times \alpha$ matrices $)$

(2) Symplectic: $A_{n}, 4 \leq n<\infty$ (antisymmetric $n \times n$ matrices) 
(3) Hermitian: $S_{n}, 2 \leq n<\infty$ (symmetric $n \times n$ matrices)

(4) Spin: $V_{\lambda}, 2 \leq \lambda\left(\operatorname{dim}\left(V_{\lambda}\right)=\lambda+1\right)$

In (1) and (4), the cardinals $\alpha$ and $\lambda$ can be infinite. $M_{n, \alpha}$, for $n \geq 1$, and $A_{2 n}, A_{2 n+1}, S_{n}$ for $n \geq 2$ are all of rank $n$. Spin factors have rank 2 and are, together with $A_{2 n}$ and $S_{n}$ for $n \geq 2$, isometric to JC*-algebras. We have the isomorphisms $S_{2} \approx V_{2}, M_{2,2} \approx V_{3}, A_{4} \approx V_{5}$ and, for $n \geq 2$, $A_{2 n} \approx M_{n}(\mathrm{H})_{\mathrm{sa}} \otimes_{\mathrm{R}} \mathrm{C}, S_{n} \approx M_{n}(\mathrm{R})_{\mathrm{sa}} \otimes_{\mathrm{R}} \mathrm{C}$. The factors $M_{1, \alpha}$ are the $\alpha$-dimensional Hilbert spaces.

There are two exceptional factors.

(5) $B_{1,2}:(1 \times 2$ matrices over the complex Cayley numbers $)$

(6) $M_{3}^{8}$ : (self-adjoint $3 \times 3$ matrices over the complex Cayley numbers)

Let $A$ be a $\mathrm{JB}^{*}$-triple. If for all $P \in \operatorname{Prim}(A), A / P$ is a finite rank rectangular Cartan factor, then $A$ is said to be of rectangular type.

The appellations symplectic, hermitian, spin and exceptional are employed correspondingly.

If for a fixed finite rank Cartan factor $M$ we have $A / P \approx M$ for all primitive ideals $P$, then $A$ is said to be of type $M$. By convention, the zero triple is considered to be of every type.

We recall that by the Gelfand-Naimark theorem of [14] in a $\mathrm{JB}^{*}$-triple $A$ there is a unique norm closed ideal $J$ such that $A / J$ is a $\mathrm{JC}^{*}$-triple and $J$ is exceptional.

Theorem 5.1. Let $A$ be a $\mathrm{JB}^{*}$-triple and let $n \in \mathrm{N}$. There is a (unique) norm closed ideal $J$ of $A$ such that $\operatorname{rank}(\pi) \leq n$ for all $\pi \in C(A / J)$ and $\operatorname{rank}(\pi)>n$ for all $\pi \in C(J)$.

Proof. This is the algebraic translation of Proposition 4.2.

COROllary 5.2. Let $A$ be a $\mathrm{JB}^{*}$-triple for which all non-exceptional Cartan factor representations have rank greater than 3 . Then the exceptional ideal of $A$ is a direct summand.

Proof. Let $J$ be the exceptional ideal of $A$. Then $h(J)=\{\operatorname{ker}(\pi)$ : $\pi \in C(A), \pi$ is non-exceptional $\}=\{\operatorname{ker}(\pi): \pi \in C(A), \operatorname{rank}(\pi)>3\}$ is both open and closed in $\operatorname{Prim}(A)$. It follows that $J$ is a direct summand.

Proposition 5.3. Let $A$ be a $\mathrm{JB}^{*}$-triple of bounded rank and let $\{\operatorname{rank}(A / P): P \in \operatorname{Prim}(A)\}=\left\{n_{1}\right\}_{i=1}^{k}$ where $1 \leq n_{1}<n_{2}<\ldots<n_{k}$. Then there is a finite composition series of norm closed ideals, $0=J_{0} \subset J_{1} \subset \ldots \subset J_{k-1} \subset J_{k}=A$ such that for $r=0, \ldots, k-1, J_{r+1} / J_{r}$ is non-trivial of constant rank $n_{k-r}$ with Hausdorff structure space.

Proof. This follows from Theorem 5.1 together with Lemma 4.4. 
Corollary 5.4. Let $A$ be an exceptional JB*-triple. Then there is a norm closed ideal $J$ of $A$ such that $J$ is of type $M_{3}^{8}$ and $A / J$ is type $B_{1,2}$.

REMARK 5.5. Let $\left(e_{i j}\right)$ be the canonical matrix units of $M_{n, \alpha}$, where $n \leq \alpha<\infty$.

(a) The tripotents $e_{11}, \ldots, e_{1 \alpha}$ form a collinear system (see Section 4 ) in $M_{n, \alpha}$. Moreover, any collinear system $S$ has cardinality $\leq \alpha$. Indeed, as two minimal tripotents are exchanged by some automorphism (see [24, §5]) we may suppose that $e_{11} \in S$. Then the collinearity and minimality of the elements of $S$ implies by straightforward calculation that $S$ is contained either in the linear span of $e_{11}, \ldots, e_{1 \alpha}$ or $S$ is contained in the linear span of $e_{11}, \ldots, e_{n 1}$. By $[8$, Lemma on page 306] it follows that card $(S) \leq \alpha$.

(b) Let $n \geq 4$ and $f_{i j}=e_{i j}-e_{j i}, 1 \leq i, j \leq n$. Then $\left\{f_{12}, \ldots, f_{1 n}\right\}$ is a collinear system in $A-n$. Let $S$ be any collinear system in $A_{n}$. The claim now is that $\operatorname{card}(S) \leq n-1$. As before, by $[24, \S 5]$, we may suppose that $f_{12} \in S$. In this case calculation shows that $S \backslash\left\{f_{12}\right\}$ is contained in the image of the injective triple homomorphism $\pi: M_{2, n-2} \rightarrow A_{n}$ given by $\pi(x)=\left[\begin{array}{cc}0 & x \\ -x^{T} & 0\end{array}\right]$. So, the claim follows from (a).

By the above results the study of $\mathrm{JB}^{*}$-triples of bounded rank reduces to that of constant rank. We shall now proceed to analyse $\mathrm{JB}^{*}$-triples of constant rank.

The main decomposition result is Theorem 5.8. The $\mathrm{JB}^{*}$-algebra version is known (cf. [6]), of which we shall make esssential use (in Lemma 5.7). In order to treat $\mathrm{JB}^{*}$-triples we need to come to grips with and synthesise phenomena that do not arise in $\mathrm{JB}^{*}$-algebras.

Lemma 5.6. Let $A$ be a $\mathrm{JB}^{*}$-triple of constant rank $n$.

(i) If $A$ is rectangular and $1 \leq n \leq \alpha<\infty$, then there is a norm closed ideal $J$ of $A$ such that all primitive quotients of $J$ and $A / J$ are respectively of the form $M_{n . \beta}$ where $\alpha<\beta \leq \infty$ and $M_{n, \beta}$ where $n \leq \beta \leq \alpha$.

(ii) If (up to isometry) $\{A / P: P \in \operatorname{Prim}(A)\}=\left\{M_{n, \alpha_{i}}\right\}_{i=1}^{k}$ where $1 \leq n \leq \alpha_{1}<\ldots<\alpha_{k}<\infty$, then there are norm closed ideals in $A, 0=J_{0} \subset J_{1} \subset \ldots \subset J_{k} \subset J_{k+1}=A$, such that $J_{r+1} / J_{r}$ is non-trivial type $M_{n, \alpha_{k-r}}$ for $r=0, \ldots, k$.

(iii) If $n \geq 2$ and $A$ is symplectic, then there is a norm closed ideal $J$ of $A$ such that $J$ is type $A_{2 n+1}$ and $A / J$ is type $A_{2 n}$.

Proof. (i) If $A$ is rectangular, then the set

$$
C_{\alpha}(A)=\left\{P \in \operatorname{prim}(A): A / P \approx M_{n, \beta}, \beta \leq \alpha\right\}
$$

is closed in $\operatorname{Prim}(A)$ by Proposition 4.7 together with Remark 5.5 (a). 
Thus $k\left(\mathrm{C}_{\alpha}(A)\right)$ is the required ideal.

(ii) This follows from (i) by repeated application.

(iii) In this case, by Proposition 4.7 and Remark 5.5 (b),

$$
S=\left\{P \in \operatorname{Prim}(A): A / P \approx A_{2 n}\right\}
$$

is closed in $\operatorname{Prim}(A)$ and $J=k(S)$ is the required ideal.

Lemma 5.7. Let $A$ be a $\mathrm{JC}^{*}$-algebra of constant rank $n$ where $3 \leq n<\infty$. Then there are norm closed ideals of $A, J_{1} \subset J_{2}$ such that $J_{1}$ is of type $A_{2 n}, J_{2} / J_{1}$ is type $M_{n, n}$ and $A / J_{2}$ is type $S_{n}$.

Proof. As all Type I factor representations (in the sense of 15, page 133) of the JC-algebra $A_{\mathrm{sa}}$ must be of Type $I_{n}$, this follows from [6, §5] because $A_{2 n} \approx M_{n}(\mathrm{H})_{\mathrm{sa}} \otimes_{\mathrm{R}} \mathrm{C}$ and $S_{2 n} \approx M_{n}(\mathrm{R})_{\mathrm{sa}} \otimes_{\mathrm{R}} \mathrm{C}$.

TheORem 5.8. Let $A$ be a $\mathrm{JC}^{*}$-triple of constant rank $n$, where $3 \leq n<\infty$. Then there are norm closed ideals of $A, J_{1} \subset J_{2} \subset J_{3}$ such that

(i) $J_{1}$ is type $A_{2 n+1}$;

(ii) $J_{2} / J_{1}$ is type $A_{2 n}$;

(iii) $J_{3} / J_{2}$ is rectangular;

(iv) $A / J_{3}$ is type $S_{n}$.

Proof. Let $P_{0} \in \operatorname{Prim}(A)$. Let $x \in A$ and $e$ be complete tripotent of $M=A / P_{0}$ such that $x+P_{0}=e$. Then by Section $2, A(x) / P_{0} \cap A(x) \approx M_{2}(e)$ as $\mathrm{JC}^{*}$-algebras. Let $I$ be the norm closed ideal of the JC*-algebra $A(x)$ such that

$$
V=\operatorname{Prim}(A(x)) \backslash h(I)=\{Q: \operatorname{rank}(A(x) / Q)=n\} .
$$

Then $P_{0} \cap A(x) \in V$ and $I$ is a $\mathrm{JC}^{*}$-algebra of constant rank $n$.

Now suppose that $P_{0} \in S=\{P \in \operatorname{Prim}(A): A / P$ is symplecic $\}$. Then $M \approx A_{2 n}$ or $A_{2 n+1}$ so that $M_{2}(e) \approx A_{2 n}$, and by Lemma 5.7 there is a nonzero norm closed ideal $J$ of $I$ all primitive quotients of which are isometric to $A_{2 n}$. Then $P_{0} \cap J \neq 0$. Let $K=T(J)$ be the norm closed ideal of $A$ generated by $J$ and let $P \in \operatorname{Prim}(A)$ such that $P \cap K \neq 0$. Then $P \cap J \neq 0$, by Lemma 2.4. Hence, $A_{2 n} \approx J / P \cap J$ imbeds as a subtriple into $A / P$. As $A_{2 n}$ cannot be so embedded into $M_{n, \alpha}$ nor into $S_{n}$, we must have $A / P \approx A_{2 n}$ or $A_{2 n+1}$. Therefore, $P \in \operatorname{Prim}(A) \backslash h(K) \subset S$, which proves that $S$ is open in $\operatorname{Prim}(A)$. Hence, there is a norm closed ideal $J_{2}$ of $A$ such that $J_{2}$ is symplectic and $A / J_{2}$ has no symplectic primitive quotients. The required ideal $J_{1} \subset J_{2}$ comes from Lemma 5.6 (iii).

Passing to $A / J_{2}$ we may assume that $J_{2}=0$ and emulate the above argument for $P_{0} \in R=\{P \in \operatorname{Prim}(A): A / P$ is rectangular $\}$. In this case, in the notations of the first paragraph of the proof, $A(x) / P_{0} \cap A(x) \approx M_{2}(e) \approx$ 
$M_{n, n}$ as $\mathrm{JC}^{*}$-algebras. Applying Lemma 5.7 together with the fact that $M_{n, n}$ is not embeddable in $S_{n}$, we obtain by the same argument an ideal $J_{3}$ of $A$ such that $J_{3}$ is rectangular and $A / J_{3}$ has no rectangular primitive quotients and so is type $S_{n}$.

It remains to deal with the general constant rank 2 case (Lemma 5.6(i) takes care of the general constant rank 1 case). Let $V \subset M$, where $V$ is a spin factor and $M$ is a $\mathrm{JBW}^{*}$-triple factor of rank 2 . For convenience we tabulate the possible structure of $M$ determined by $V=V_{\alpha}, \alpha \geq 3$.

\begin{tabular}{c|c|c|c|c}
$V$ & $V_{3}$ & $V_{4}$ & $V_{5}$ & $V_{\alpha>5}$ \\
\hline$M$ & $M_{2, \alpha}, A_{5}, V_{\gamma \geq 3}$ & $A_{5}, V_{\gamma \geq 4}$ & $A_{5}, V_{\gamma \geq 5}$ & $V_{\gamma>5}$
\end{tabular}

TheORem 5.9. Let $A$ be a $\mathrm{JC}^{*}$-triple of constant rank 2. If $A$ is a spin type and $2 \leq \gamma<\infty$, then $S^{\gamma}(A)=\left\{P \in \operatorname{Prim}(A): A / P \approx V_{\lambda}, \lambda>\gamma\right.$ is open in $\operatorname{Prim}(A)$.

In general, there are ideals $J_{1} \subset J_{2} \subset J_{3} \subset J_{4} \subset J_{5} \subset A$ such that

(i) $J_{1}$ is spin type with $\operatorname{Prim}\left(J_{1}\right)=S^{5}$;

(ii) $J_{2} / J_{1}$ is type $A_{5}$;

(iii) $J_{3} / J_{2}$ is type $V_{5}$;

(iv) $J_{3} / J_{2}$ is type $V_{4}$;

(v) $J_{5} / J_{4}$ is rectangular;

(vi) $A / J_{5}$ is type $V_{2}$.

Proof. Let $P_{0} \in(A)$. As in the proof of Theorem 5.8, for a complete tripotent $e \in M$ and $x \in A$ we have $A(x) / P_{0} \cap A(x) \approx M_{2}(e)$ as $\mathrm{JC}^{*}$-algebras. As $M$ is rank $2, M_{2}(e)$ is a spin factor.

Assume that $A$ is of spin type. Suppose that $P_{0} \cap A(x) \in S^{\gamma}(A(x))$ which is open in $\operatorname{Prim}(A)$ by Lemma 4.9. Thus, by Proposition 3.3 and its notation $U=\beta^{-1}\left(S^{\gamma}(A(x))\right)$ is open neighbourhood of $P_{0}$ and $U \subset S^{\gamma}(A)$ by the table above. It follows that $S^{\gamma}(A)$ is open.

Reverting to the general case, the same argument shows that $S^{5}(A)$ is open. This gives the ideal $J_{1}$. Passing to $A / J_{1}$ we may assume that $S^{5}(A)=\phi$. In this case, suppose that $P_{0} \in\{Q \in \operatorname{Prim}(A): A / Q$ is symplectic $\}=S$. Then $M \approx A_{5}$ or $M \approx V_{5} \approx A_{4}$ so that $M_{2}(e) \approx V_{5}$ and $P_{0} \cap A(x) \in S^{4}(A(x))$. Hence, by Proposition 3.3 and Lemma 4.9 together with the above table, there is a neighbourhood of $P_{0}$ contained in $S$ which is therefore open in $\operatorname{Prim}(A)$. By Lemma 5.6(iii), the corresponding ideal, $J_{3}$ contains the ideal $J_{2}$ as stated.

Proceeding, we now assume that $S$ and $S^{5}(A)$ are empty to find, in the same way, that $S^{3}(A)$ is now open. This gives the ideal $J_{4}$.

Finally assume that $S^{3}(A)$ is empty and let 


$$
P_{0} \in R=\{P \in \operatorname{Prim}(A): A / P \text { is rectangular }\} .
$$

Then $M_{2}(e)=V_{3}$ and we find $P_{0} \in \beta^{-1}\left(S^{2}(A(x))\right) \cap R$ from the first column of the table. It follows that $R$ is open in $\operatorname{Prim}(A)$, which gives the ideal $J_{5}$.

Corollary 5.10. Let $A$ be a $\mathrm{JB}^{*}$-triple of bounded rank such that all primitive quotients are finite dimensional and let, up to isometry, $\{A / P: P \in \operatorname{Prim}(A)\}=\left\{M_{i}\right\}_{i=1}^{K}$. Then there is a permutation $\pi$ of $\{1, \ldots, k\}$ and norm closed ideals of $A, 0=I_{0} \subset I_{1} \subset \ldots I_{k} \subset I_{k+1}=A$ such that $I_{r+1} / I_{r}$ is non-trivial type $M_{\pi(r)}$, for $r=0, \ldots, k$.

Acknowledgement. The authors are grateful for financial support from the British Council and the Slovene Ministry of Science and Technology.

\section{REFERENCES}

1. E.M. Alfsen and E.G. Effros, Structure in real Banach spaces I, II, Ann. of Math. 96 (1972), 98-128 and 129-173.

2. T. Barton and R. Timoney, Weak* continuity of Jordan triple product and applications, Math Scand. 59 (1986), 177-191.

3. T. Barton, T. Dang and G. Horn, Normal representations of Banach triple systems, Proc. Amer. Math. Soc. 102 (1987), 551-555.

4. E. Behrens, M-structure and the Banach-Stone Theorem, Lectue Notes in Math. 736, 1979.

5. H. Behncke and W. Bos, JB-algebras with an exceptional ideal, Math. Scand. 42 (1978), 306312.

6. L.J. Bunce Structure of representations and ideals of homogeneous type in Jordan algebras, Quart. J. Math. Oxford 37 (1986), 1-10.

7. L.J. Bunce and C.H. Chu, Compact operations, multipliers and the Radon- Nikodym property, Pacific J. Math 153 (1992), 249-265.

8. T. Dang and Y. Friedman, Classification of $J B W^{*}$-triple factors and applications, Math. Scand. 61 (1987), 292-330.

9. S. Dineen, The second dual of a JB*-triple system, Complex Analysis, Functional Analysis and Approximation theory, vol. 125, North Holland Math. Studies, 1986, pp. 67-69.

10. J. Dixmier, $C^{*}$-algebras, North Holland, 1969.

11. C.M. Edwards, On the centres of hereditary JBW subalgebras of a JBW algebra, Math. Proc. Cambridge Philos. Soc. 85 (1979), 317-324.

12. Y. Friedman and B. Russo, Structure of the predual of a $J B W^{*}$-triple, J. Reine Angew. Math. 356 (1985), 67-84.

13. Y. Friedman and B. Russo, Solution of the contractive projection problem, J. Funct. Anal. 60 (1985), 56-79.

14. Y. Friedman and B. Russo, The Gelfand-Naimark theorem for JB*triples, Duke Math. J. 53 (1986), 139-148.

15. H. Hanche-Olsen and E. Stormer, Jordan Operator Algebras, Pitman, London 1984.

16. P. Harmand, D. Werner and W. Werner, M-ideals in Banach Spaces and Banach algebras. Lecture Notes in Math. 1547, 1993.

17. L.A. Harris, A generalization of $C^{*}$-algebras, Proc. London Math. Soc. 42 (1982), 331-361.

18. G. Horn, Characterization of the predual and ideal structure of a $J B W^{*}$ triple, Math. Scand. 61 (1987), 117-133.

19. G. Horn, Classification of JBW*-triples of type I, Math. Z. 196 (1987), 271-291. 
20. E. Horn and E Neher, Classification of $J B W^{*}$-triples, Trans. Amer. Math. Soc. 306 (1988), 553-578.

21. W. Kaup, A Riemann mapping theorem for bounded symmetric domains in complex Banach spaces, Math. Z. 138 (1983), 503-529.

22. W. Kaup, Contractive projections on Jordan $C^{*}$-algebras and generalizations, Math. Scand. 54 (1985), 95-100.

23. W. Kaup, On JB*-triples defined by fibre bundles, Manuscripta Math. 87 (1987), 379-403.

24. O. Loos, Bounded Symmetric Domains and Jordan Pairs (1977), Irvine Lecture Notes.

25. G.K. Pedersen, $C^{*}$-algebras and their automorphism groups, North Holland, 1979.

26. B. Russo, Structure of JB*triples, Jordan Algebras (W. Kaup, K. McCrimmon, H.P. Petterson, eds.), de Gruyter, 1994.

27. L.L. Stacho, A projection principle concerning biholomorphic automorphisms of Banach spaces, Acta Sci. Math. (Szeged) 46 (1982).

28. J. Tomiyama and M.Takesaki, Application of fibre bundles to the certain class of $C^{*}$-algebras, Tohoku Math. J. 13 (1961), 498-522.

29. H. Upmeier, Symmetric Banach Manifolds and Jordan $C^{*}$-algebras, North Holland, 1985.

30. J.D.M Wright, Jordan $C^{*}$-algebras, Michigan Math. J. 24 (1977), 291-302.

THE UNIVERSITY OF READING

DEPARTMENT OF MATHEMATICS

WHITEKNIGHTS

READING RG6 6AX

UK

UNIVERSITY OF LONDON

GOLDSMITH'S COLLEGE

NEW CROSS

LONDON SE14 6NW

UK

Email address: maa01che@gold.ac.uk

UNIVERSITY OF MARIBOR

FACULTY OF CIVIL ENGINEERING

DEPARTMENT OF BASIC SCIENCES

SMETANOVA 17

62000 MARIBOR

SLOVENIJA

E-mail address: Borut.Zalar@uni-mb.si or borut.zalar@uni-lj.si 